\title{
Pharmaceutical Regulation: Crossroad of Opportunity as the Distinction between Food and Drugs Blurs
}

Elizabeth A. Perry ${ }^{1}$, Rudolph J. Castellani² and George Perry ${ }^{1 *}$

${ }^{1}$ Department of Biology, University of Texas at San Antonio, San Antonio, Texas, USA

${ }^{2}$ Department of Pathology, University of Maryland Baltimore, Baltimore, Maryland, USA

\section{Editorial}

Much attention has been drawn to impending patent expiration of several highly marketed drugs and the action of pharmaceutical companies to skirt investment through consolidation or changes in dosing/marketing. Overlooked is what promises to be a larger movement: the slow transformation in understanding the largest market for drugs, those that address chronic age-related disease. Increased realization of these conditions as long-term adaptive survival responses to the aging process has revealed the critical role of lifestyle, whether the conditionis cancer, heart disease, arthritis, diabetes, or neurodegenerative disease. Socioeconomic factors, such as education, exercise, and nutrient intake, have emerged as primary determinants that are incompletely addressed by drugs alone. Lifestyle control has played a major part in reduction of heart disease and cancer, and lack of control in other aspects is responsible for increased diabetes displaying as metabolic syndrome. Aggressive development of approaches to exploit natural adaptive responses is being used to promote health. The market for nutraceuticals and medical foods is growing at a rapid rate, $6-7 \%$ growth, with weak oversight, based on the belief that these products' derivation from food or other natural sources makes them 'safe'. Overlooked is that many well known drugs were similarly derived.
Both food industry and pharmaceutical giants are entering this market, now estimated at US $\$ 151$ Billion annually. As development proceeds, it is critical to assess safety no matter what the origin. Why? Because in the natural world, plants and diverse animals accumulate complex chemical compounds not just to function, but also to protect themselves from predators. It may well be that the health benefits of vegetables and fruits derive as much from these 'toxins' as vitamins, evidenced by the weak record of vitamin supplements to augment health of well nourished individuals. Further, even the 'safest' of foods underlie the biggest health problem we are now facing, metabolic syndrome. We do not know if metabolic syndrome is a result of a high carbohydrate diet, lack of exercise, or other changes. An understanding of the mechanism of action of these supplements and diet alterations is essential as diet and nutraceuticals are used to promote health. Looking inward to nutrients and health looks back to the origins of medicinal practice where plant extracts and diet were the primary treatments, but can now be enriched by modern empirical clinical trials to demonstrate efficacy and safety. The application of modern research techniques to traditional medicine may be the answer to today's largest health concerns.
*Corresponding author: George Perry, Department of Biology, University of Texas at San Antonio, San Antonio, Texas, USA, Tel: 210-458-4450; Fax: 210458-4445; Email: george.perry@utsa.edu

Received October 30, 2012; Accepted October 31 2012; Published November 02, 2012

Citation: Perry EA, Castellani RJ, Perry G (2012) Pharmaceutical Regulation: Crossroad of Opportunity as the Distinction between Food and Drugs Blurs. Pharmaceut Reg Affairs 1:e117. doi:10.4172/2167-7689.1000e117

Copyright: (C) 2012 Perry EA, et al. This is an open-access article distributed under the terms of the Creative Commons Attribution License, which permits unrestricted use, distribution, and reproduction in any medium, provided the original author and source are credited. 